\title{
Relationship between AdeABC Efflux Pump Genes and Carbapenem in Multidrug-resistant Acinetobacter baumannii
}

\author{
Yeongdon Ju ${ }^{1,2, \$, *}$, Yoo-Jeong Kim ${ }^{1, \S, *}$, Chulhun L. Chang ${ }^{3, * *}$, \\ Go-Eun Choi ${ }^{1, * *}$ and Kyung-Yae Hyun ${ }^{4, ;}, * *$ \\ ${ }^{I}$ Department of Clinical Laboratory Science, College of Health Sciences, \\ Catholic University of Pusan, Busan 46252, Korea \\ ${ }^{2}$ Clinical Trial Specialist Program for In Vitro Diagnostics, Brain Busan 21 Plus Program, \\ the Graduate School, Catholic University of Pusan, Busan 46252, Korea \\ ${ }^{3}$ Department of Laboratory Medicine, Pusan National University Yangsan Hospital, Yangsan 50612, Korea \\ ${ }^{4}$ Department of Clinical Laboratory Science, Dong-Eui University, Busan 47340, Korea
}

\begin{abstract}
Multidrug-resistant strain of Acinetobacter baumannii (MDRAB) is an emerging pathogen in health care facilities, preventing MDRAB is a public health concern. We conducted this experiment on a clinical isolate of A. baumannii with two main goals: the role of the efflux pump system in the stress provision of carbapenem and the response to the transcription level of the efflux pump gene. A total of 34 strains of A. baumannii was isolated from the Yangsan Hospital of Pusan National University. First, when we compared and observed the expression of the efflux pump gene and antibacterial resistance to carbapenem, a strong correlation was observed between carbapenem resistance and overexpression of adeB ( $P=0.0056$ ). Second, a correlation between the efflux pump and concentration gradient and tolerance to carbapenem stress at the $A d e A B C$ efflux pump genes transcription level was confirmed. Our results revealed that the expression of the $A d e A B C$ efflux pump is an important resistance determinant in obtaining antibiotic resistance of the carbapenem group in A. baumannii.
\end{abstract}

Key Words: Acinetobacter baumannii; Carbapenem; Drug-resistance; Efflux pumps; AdeABC; adeB

\section{INTRODUCTION}

Multidrug-resistant Acinetobacter baumannii (MDRAB) is an emerging pathogen in health care facilities, especially in the intensive care unit. It has been involved in nosocomial infections and a number of hospital outbreaks. Multi-drug resistance, defined as resistance to three or more antimicrobial classes including carbapenems (such as imipenem and meropenem), aminoglycosides (such as amikacin and gentamicin), and fluoroquinolones (such as ciprofloxacin and levofloxacin) has increased over the past decade (Batirelet al., 2014; Pogue et al., 2014). In the antimicrobial susceptibility studies of $A$. baumannii isolated from Korean adults, the resistance rates against $\beta$-lactam, aminoglycoside, and fluoroquinolone antibiotics increased from $55 \%, 48 \%$, and $67 \%$ in 2009 to $86 \%, 60 \%$, and $87 \%$ in 2015 , respectively. In particular, the resistance to carbapenem increased from

Received: May 24, 2021 / Revised: June 25, 2021 / Accepted: June 28, 2021

*Graduate student, ${ }^{* *}$ Professor.

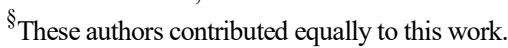

${ }^{\dagger}$ Corresponding author: Kyung-Yae Hyun. Department of Clinical Laboratory Science, Dong-Eui University, Busan 47340, Korea.

Tel: +82-51-890-2683, Fax:+82-0505-182-6877, e-mail: kyhyun@deu.ac.kr

(C) The Korean Society for Biomedical Laboratory Sciences. All rights reserved.

(C) This is an Open Access article distributed under the terms of the Creative Commons Attribution Non-Commercial License (http://creativecommons.org/licenses/by-nc/3.0/) which permits unrestricted non-commercial use, distribution, and reproduction in any medium, provided the original work is properly cited. 
$15 \sim 30 \%$ in 2004 to $80 \sim 85 \%$ in 2014 (Bae et al., 2012; Park et al., 2018; Kim and Park, 2018). For the treatment of A. baumannii, colistin is selected as the primary treatment; alternatively, a combination antimicrobial therapy consisting aminoglycoside or fluoroquinolone may be considered in cases where the required minimum inhibitory concentration (MIC) of meropenem is greater than $8 \mu \mathrm{g} / \mathrm{mL}$. For $A$. baumannii strains that are resistant to both aminoglycoside and fluoroquinolone, a combination therapy of the antimicrobial agents fosfomycin, ampicillin/sulbactam, or tigecycline is recommended, depending on the susceptibility profile (Hsu and Tamma, 2014).

There are various mechanisms of antibiotic resistance in A. baumannii, such as the production of carbapenemases, overexpression of efflux pumps, genetic alterations of penicillin binding proteins and loss of porins expression (RosalesReyes et al., 2017). Efflux pump activation due to overexpression of related genes, one of the major mechanisms in MDRAB (Lin et al., 2017), release unwanted or harmful substances (antibiotics, neurotransmitters, and toxic substances) out of cells through specific transporters in the cell membrane (Xing et al., 2014). It is well known that efflux pumps are one of the most important systems in bacteria responsible for both congenital and acquired antimicrobial resistance (Blair et al., 2015). Over-expression of efflux pumps and their related genes have been reported to contribute to multidrug resistance in $P$. aeruginosa (Shigemura et al., 2015). Several studies have demonstrated the role of efflux pumps in fluoroquinolone resistant $E$. coli (AmabileCuevas et al., 2010; Swick et al., 2011). Similarly, fluoroquinolone resistant clinical isolates of Shigella flexneri and Shigella sonnei showed overexpression of the TolC channels, which are part of AcrAB-TolC tripartite, in response to ciprofloxacin (Kim et al., 2008). Several studies have demonstrated that the up-regulation of efflux pump genes contribute significantly to decreasing the intracellular antibiotic concentration with a selectivity to the efflux transporter (Nikaido, 2009).

Efflux pumps have been classified into five different major superfamilies: ATP-binding cassette superfamily (ABC), small multidrug resistance family (SMR), multi antimicrobial extrusion protein family (MATE), major facilitator super- family (MFS), and resistance-nodulation-cell division superfamily (RND) (Fernandez and Hancock, 2013; Sun et al., 2014). The AcrAB efflux pump system, comprising the $m a c A, m a c B$, and tolC genes, is a member of the $\mathrm{ABC}$ superfamily that pumps out bile acids and fatty acids to promote resistance against aminoglycoside and tetracycline (Chan et al., 2015). The RND family has AdeABC, AdeIJK, and AdeFGH pumps that reduce the susceptibility to various antibiotics in A. baumannii (Xing et al., 2014). The CraA AmvA, CmlA, MdfA, and Tet pumps, part of the MFS family, have been identified in A. baumannii (Roca et al., 2009). AbeM is the only efflux pump in the MATE family toincrease the MIC of antibiotics in A. baumannii (Su et al., 2005) AbeS, part of the SMR family, is a low level resistance pump for some antibiotics in A. baumannii (Srinivasan et al., 2009).

Although efflux pump-mediated antibiotic resistance is known for aminoglycoside and tetracycline resistance, its role in carbapenem non-susceptibility has not been established in A. baumannii. As the incidence of carbapenem resistance increasing in A. baumannii (Rosales-Reyes et al., 2017), it is very important to explore the mechanism of obtaining carbapenem-related resistance. Understanding the contribution of efflux pumps to carbapenem resistance in $A$. baumamnii will enable the development of therapies targeting the rising number of MDRAB infections in Korea. Hence, in the present study, we investigated the role of efflux pumps in resistance against carbapenem antibiotics and the transcriptional response of efflux pump genes to carbapenem stress in clinical isolates of $A$. baumannii.

\section{MATERIALS AND METHODS}

\section{Bacterial strains and growth conditions}

A total of $34 \mathrm{~A}$. baumannii isolates were isolated and collected from a patient at the Pusan National University Yangsan Hospital (PNUYH), Busan, South Korea from November 4, 2011 to July 11, 2018. All of the strains were identified by the Vitek system (bioMerieux, Inc., Hazelwood, MO, USA) and collected from each patient. 


\section{Susceptibility testing}

The MIC values of all the strains to each drug were tested by the broth microdilution assay recommended by the Clinical and Laboratory Standards Institute (Wayne, 2012). Antibiotics were purchased from Sigma Chemical Company (St. Louis, MO, USA). Briefly, a 0.5 McFarland standard $\left(1.5 \times 10^{8}\right.$ colony forming units $\left./ \mathrm{mL}\right)$ bacterial suspension was prepared, diluted using MH broth (Oxoid, Basingstoke, United Kingdom) and added to the wells of a 96-well plate at different concentrations (ranging from 0.125 to $1,024 \mu \mathrm{g}$ /mL). A. baumannii ATCC17978 and Escherichia coli ATCC29522 were both used as quality control strains.

\section{Detection of efflux pump function}

The MIC values of meropenem and imipenem were detected with the two-fold agar dilution method (as recommended by the 2012 CLSI) in the presence and absence of efflux pump inhibitors to determine the contribution of efflux pumps to resistance. Mueller-Hinton ( $\mathrm{MH}$; Oxoid, Basingstoke, UK) agar containing carbonyl cyanide 3chlorophenylhydrazone (CCCP; Sigma-Aldrich, St. Louis, $\mathrm{MO}$, USA) or phenyl-arginine- $\beta$-naphthylamide (PA $\beta \mathrm{N}$; Sigma-Aldrich) was prepared. Seoul, Korea. To confirm the activity of Gram-negative bacteria on the RND pump, experiments were performed using $\mathrm{MH}$ agar supplemented with CCCP or PA $\beta N$. (Pagès et al., 2005). To evaluate the effect of $\mathrm{CCCP}$ or PA $\mathrm{N}$ on bacterial growth, all the bacteria were cultured in the $\mathrm{MH}$ agar containing the final concentration of CCCP $(25 \mu \mathrm{g} / \mathrm{mL})$ or PA $\beta \mathrm{N}(70 \mu \mathrm{g} / \mathrm{mL})$. The criteria for a positive efflux phenotype were defined as an MIC value with CCCP or PA $\beta \mathrm{N}$ that decreased 4-fold or more compared to the MIC values without the efflux pump inhibitors (Shi et al., 2005). Both the sensitive and resistant strains were used for the test, and A. baumannii ATCC17978 was selected as the quality control strain.

\section{Detection of the expression level of efflux pump genes by qPCR}

Following bacterial RNA extraction with the RNeasy Mini kit (Qiagen, Valencia, CA, USA), the total RNA was reverse transcribed into cDNA and RT-qPCR was performed using the first strand cDNA synthesis kit (Takara Biotechnology, Dalian, China) and ReverTraAce qPCR RT Master Mix (Toyobo, Osaka, Japan) according to the manufacturer's recommendations. The efflux pump genes were the target genes and 16S rRNA served as an internal reference gene (Peleg et al., 2007) (Table 1). qRT-PCR was performed in a 7500 Fast Real-Time PCR System (Applied Biosystems, Foster City, CA, USA) with SYBR ${ }^{\circledR}$ qPCR Mix (Toyobo) according to the manufacturer' recommendations. The qRTPCR assay was performed using specific primers for adeA, adeB, adeC, adeS, adeR, macB, abeM, craA, adeF, adeJ, and $16 \mathrm{~S}$ rRNA (in clinical isolates of $A$. baumannii as previously described). Primers were synthesized by Macrogen (Seoul, Korea) and primer sequences are listed in Table 1. The reaction conditions consisted of three phases: predenaturation at $95^{\circ} \mathrm{C}$ for $10 \mathrm{~min}$; 40 cycles of denaturation at $95^{\circ} \mathrm{C}$ for $10 \mathrm{~s}$, annealing at $55^{\circ} \mathrm{C}$ for $20 \mathrm{~s}$, extension at $72^{\circ} \mathrm{C}$ for $15 \mathrm{~s}$; and one cycle of dissociation at $95^{\circ} \mathrm{C}$ for 10s. Expression levels of the target and internal control genes were determined at the same time, and each assay was repeated three times. The results were analyzed by the $\Delta \Delta \mathrm{Cq}$ method (Livak and Schmittgen, 2001). One positive strain was selected as the control sample. Standardization was performed by the subtraction of the mean $\mathrm{Cq}$ value of each gene and the mean $\mathrm{Cq}$ value of the corresponding $16 \mathrm{~S}$ rRNA (repeated three times for each sample), yielding a corrected $\Delta \mathrm{Cq}$ value. Following correction, subtraction of the $\Delta \mathrm{Cq}$ value of the resistant strain from the mean $\Delta \mathrm{Cq}$ value of the positive strain yielded $\Delta \Delta \mathrm{Cq}$. Finally, the relative mRNA expression level of the relative mRNA expression level of the genes were calculated using the $2^{-\Delta \Delta \Delta C q}$ method.

\section{DNA sequence analysis}

To investigate the adeR and adeS gene sequences, the 34 A. baumannii clinical isolates were also subjected to PCR using specific primers, adeR (F: AGATGACTACGATATTGGCNAC, R: AGCGANGACTTCATTTGGGT) and adeS (F: ACCCAAATGAAGTCNTCGCT, R: GTTCGCTCTAGTGCATCGCT) (Ostad Asadolah-Malayeri et al., 2016). The amplicons were purified with a PCR purification kit (Qiagen); these purified amplicons were sequenced using a BigDye Terminator Cycle Sequencing Kit (Applied Bio- 
Table 1. The sequences of primers used for polymerase chain reaction to detect Acinetobacter baumannii efflux pump genes

\begin{tabular}{|c|c|c|}
\hline Gene & Sequence & Reference \\
\hline \multirow{2}{*}{ adeA } & F: 5'-ATC TTC CTG CAC GTG TAC AT-3' & \multirow{2}{*}{ Jassim et al., 2016} \\
\hline & R: 5'-GGC GTT CAT ACT CAC TAA CC-3' & \\
\hline \multirow{2}{*}{$a d e B$} & F: 5'-GTATGAATTGATGCTGC-3' & \multirow{2}{*}{ Jassim et al., 2016} \\
\hline & R: 5'-CACTCGTAGCCAATACC-3' & \\
\hline \multirow{2}{*}{ adeC } & F: 5'-AGCCTGCAATTACATCTCAT-3' & \multirow{2}{*}{ Jassim et al., 2016} \\
\hline & R: 5'-TGGCACTTCACTATCAATAC-3' & \\
\hline \multirow{2}{*}{ adeS } & F: 5'-TGTGGGTTATGCAGTTGCTTTT-3' & \multirow{2}{*}{ Ostad Asadolah-Malayeri et al., 2016} \\
\hline & R: 5'-GGCATAGGCAATCCCGATT-3' & \\
\hline \multirow{2}{*}{ adeR } & F: 5'-ACTACGATATTGGCGACATT-3' & \multirow{2}{*}{ Ostad Asadolah-Malayeri et al., 2016} \\
\hline & R: 5'-GCGTCAGATTAAGCAAGATT-3' & \\
\hline \multirow{2}{*}{$\operatorname{macB}$} & F: 5'-CGGAATGGGTTCGGATGAC-3 & \multirow{2}{*}{ Lin et al., 2017} \\
\hline & R: 5'-CGGCTCATGACCGTGGTATAA-3' & \\
\hline \multirow{2}{*}{$a b e M$} & F: 5'-GTAGGTGTAGGCTTATGGA -3' & \multirow{2}{*}{ Qiu et al., 2016} \\
\hline & R: 5'-GTACCGAAGTGACTGAAAT-3' & \\
\hline \multirow{2}{*}{ craA } & F: 5'-CCTGATTCAGCCAGCCATGT-3' & \multirow{2}{*}{ Lin et al., 2017} \\
\hline & R: 5'-GAAGACGGCGCCCAAGT-3' & \\
\hline \multirow{2}{*}{ adeF } & F: 5'-GGTGTCGACCAAGATAAACG-3' & \multirow{2}{*}{ Ostad Asadolah-Malayeri et al., 2016} \\
\hline & R: 5'-GTGAATTTGGCATAGGGACG-3' & \\
\hline \multirow{2}{*}{ adeJ } & F: 5'-GCGAATGGACGTATGGTTCT-3' & \multirow{2}{*}{ Ostad Asadolah-Malayeri et al., 2016} \\
\hline & R: 5'-CATTGCTTTCATGGCATCAC-3' & \\
\hline \multirow{2}{*}{ 16s rRNA } & F: 5'-AGCATTTCGGATGGGAACTTTA-3' & \multirow{2}{*}{ Lin et al., 2017} \\
\hline & R: 5'-GTCGTCCCCGCCTTCCT-3' & \\
\hline
\end{tabular}

systems) and an ABI PRISM 3730XL DNA analyzer (Applied Biosystems). The various DNA sequences were confirmed using the BLAST paired alignment functionality.

\section{Transcriptional response of AdeABC efflux pump related} genes against a carbapenem concentration gradient

Transcriptional levels were also determined after exposing the 34 A. baumannii clinical isolates at different subinhibitory concentrations of meropenem (Sigma-Aldrich) and imipenem (Sigma-Aldrich) ranging from $0.25 \mu \mathrm{g} / \mathrm{mL}$ up to $2 \mu \mathrm{g} / \mathrm{mL}$ by qRT-PCR. Each sample was processed in triplicate and their relative expression was compared with that of A. baumannii ATCC17978 without antibiotic stress.

\section{Statistical analysis}

SPSS 17.0 (SPSS Inc., Chicago, IL, USA) and GraphPad Prism v. 5.01 (GraphPad Software Inc., San Diego, CA,
USA) were used for all statistical analyses. Comparative analyses were executed by $\chi^{2}$ or Fisher exact tests for categorical variables and by the Student $t$ test for continuous variables. All tests were two-tailed, and $P$ values of $<0.05$ are considered statistically significant.

\section{RESULTS}

\section{Antimicrobial susceptibility}

Susceptibility profiles of CNAB (Carbapenem-Nonsusceptible A. baumannii) and CSAB (Carbapenem-Susceptible A. baumannii) isolates are presented in Table 2. All these $A$. baumannii isolates were multi-drug resistant, and they showed sufficient susceptibility only to minocycline (94.1\%) and colistin (82.3\%). With regard to piperacillin, all isolates of A. baumannii showed high-level resistance, with MICs ranging from 128 to $\geq 1,024 \mu \mathrm{g} / \mathrm{mL}$, with an $\mathrm{MIC}_{50}$ of 512 
Table 2. Susceptibility profiles of $29 \mathrm{CNAB}$ and $5 \mathrm{CSAB}$ isolates

\begin{tabular}{|c|c|c|c|c|c|c|c|c|c|}
\hline \multirow[b]{2}{*}{ Drug } & \multicolumn{2}{|c|}{ MIC range $(\mu \mathrm{g} / \mathrm{mL})$} & \multicolumn{2}{|c|}{$\mathrm{MIC}_{50}(\mu \mathrm{g} / \mathrm{mL})$} & \multicolumn{2}{|c|}{$\mathrm{MIC}_{90}(\mu \mathrm{g} / \mathrm{mL})$} & \multicolumn{3}{|c|}{ No. (\%) of susceptible isolates } \\
\hline & $\begin{array}{l}\text { CNAB } \\
(\mathrm{n}=29)\end{array}$ & $\begin{array}{c}\mathrm{CSAB} \\
(\mathrm{n}=5)\end{array}$ & $\begin{array}{l}\text { CNAB } \\
(\mathrm{n}=29)\end{array}$ & $\begin{array}{l}\mathrm{CSAB} \\
(\mathrm{n}=5)\end{array}$ & $\begin{array}{l}\text { CNAB } \\
(\mathrm{n}=29)\end{array}$ & $\begin{array}{l}\text { CSAB } \\
(\mathrm{n}=5)\end{array}$ & $\begin{array}{c}\text { All isolates } \\
(\mathrm{n}=34)\end{array}$ & $\begin{array}{l}\text { CNAB } \\
(\mathrm{n}=29)\end{array}$ & $\begin{array}{l}\text { CSAB } \\
(\mathrm{n}=5)\end{array}$ \\
\hline Meropenem & $16 \sim 128$ & $0.5 \sim 4$ & 128 & 1 & 256 & 4 & 5 & $0(0)$ & $5(100)$ \\
\hline Imipenem & $32 \sim 256$ & $1 \sim 4$ & 128 & 2 & 256 & 4 & 5 & $0(0)$ & $5(100)$ \\
\hline Ceftazidime & $32 \sim \geq 1,024$ & $32 \sim 512$ & 128 & 128 & 512 & 128 & 0 & & \\
\hline Cefepime & $32 \sim 256$ & $32 \sim 256$ & 128 & 64 & 256 & 128 & 0 & & \\
\hline Cefotaxime & $16 \sim 128$ & $16 \sim 64$ & 64 & 32 & 128 & 64 & 0 & & \\
\hline $\begin{array}{l}\text { Ampicillin/ } \\
\text { sulbactam }\end{array}$ & $16 \sim 256$ & $16 \sim 64$ & 64 & 32 & 128 & 64 & 4 & $1(3.4)$ & $3(60)$ \\
\hline Minocycline & $1 \sim 32$ & $0.5 \sim 4$ & 16 & 2 & 32 & 1 & 32 & $27(93.1)$ & $5(100)$ \\
\hline Gentamicin & $16 \sim \geq 1,024$ & $2 \sim 64$ & 128 & 16 & 512 & 64 & 2 & 0 & $2(40)$ \\
\hline Ciprofloxacin & $4 \sim 128$ & $4 \sim 64$ & 64 & 32 & 128 & 64 & 0 & & \\
\hline Tigecycline & $0.5 \sim 32$ & $0.5 \sim 4$ & 8 & 1 & 16 & 4 & 12 & $8(27.5)$ & $4(80)$ \\
\hline Piperacillin & $128 \sim \geq 1,024$ & $16 \sim \geq 1,024$ & 512 & 512 & $\geq 1,024$ & $\geq 1,024$ & 2 & 0 & $2(100)$ \\
\hline Colistin & $0.5 \sim 4$ & $0.5 \sim 1$ & 1 & 1 & 1 & 1 & 28 & $23(79.3)$ & $5(100)$ \\
\hline
\end{tabular}

Table 3. Reduction in MIC after addition of efflux pump inhibitors

\begin{tabular}{|c|c|c|c|c|}
\hline \multirow{2}{*}{ Drug } & \multicolumn{2}{|c|}{ No. $(\%)$ of isolates with $\geq 4$-fold reduction in MIC } & \multicolumn{2}{|c|}{ No. $(\%)$ of isolates with $\geq 8$-fold reduction in MIC } \\
\hline & CNAB $(n=29)$ & $\operatorname{CSAB}(n=5)$ & CNAB $(n=29)$ & $\operatorname{CSAB}(n=5)$ \\
\hline Meropenem + CCCP & 9 & 2 & 7 & 2 \\
\hline Meropenem + PA $\beta N$ & 6 & 2 & 3 & 2 \\
\hline Imipenem + CCCP & 10 & 3 & 8 & 2 \\
\hline Imipenem + PA $\beta N$ & 8 & 2 & 6 & 1 \\
\hline
\end{tabular}

CNAB, carbapenem-nonsusceptible A. baumannii; CSAB, carbapenem-susceptible A. baumannii; CCCP, carbonyl cyanide 3chlorophenylhydrazone; PA $\beta \mathrm{N}$, phenyl-arginine- $\beta$-naphthylamide

$\mu \mathrm{g} / \mathrm{mL}$ and an $\mathrm{MIC}_{90}$ of $\geq 1,024 \mu \mathrm{g} / \mathrm{mL}$ as well. Of the 34 clinical A. baumannii isolates, only 12 isolates showed susceptibility to tigecycline. However, CNAB exhibited a much lower drug susceptibility phenotype than CSAB. CNAB isolates displayed significantly lower susceptibility rates to ampicillin/sulbactam $(3.4 \%$ for $\mathrm{CNAB}$ versus $60 \%$ for $\mathrm{CSAB}$ ) and gentamicin ( $0 \%$ for CNAB versus $40 \%$ for CSAB).

\section{Efflux mechanism}

All isolates grew well in the presence of $25 \mu \mathrm{g} / \mathrm{mL}$ CCCP or $70 \mu \mathrm{g} / \mathrm{mL}$ PA $\beta \mathrm{N}$. The effects of the efflux pump inhibitors on carbapenem MICs are shown in Table 3. When $25 \mu \mathrm{g} /$ $\mathrm{mL} \mathrm{CCCP} \mathrm{was} \mathrm{present,} \mathrm{4-} \mathrm{to} \mathrm{8-fold} \mathrm{decreases} \mathrm{in} \mathrm{the} \mathrm{MIC} \mathrm{of}$ imipenem were observed in $18 \mathrm{CNAB}$ isolates and $5 \mathrm{CSAB}$ isolates. Addition of $25 \mu \mathrm{g} / \mathrm{mL}$ CCCP led to 4 - to 8 -fold reductions of the meropenem MICs in $16 \mathrm{CNAB}$ isolates and $4 \mathrm{CSAB}$ isolates. After exposure to $70 \mu \mathrm{g} / \mathrm{mL} \mathrm{PA} \beta \mathrm{N}$, a 4- to 8-fold decrease of the imipenem MICs occurred in 14 CNAB isolates and $3 \mathrm{CSAB}$ isolates. A 4- to 8-fold decrease of the meropenem MICs was observed in nine CNAB isolates and four CSAB isolates after the addition of $70 \mu \mathrm{g} /$ mL PA $\beta$ N.

\section{Expression of efflux pump genes}

The expression of genes encoding efflux pump components in CNAB and CSAB isolates are presented in Fig. 1. When we compared the expression of efflux pump genes 

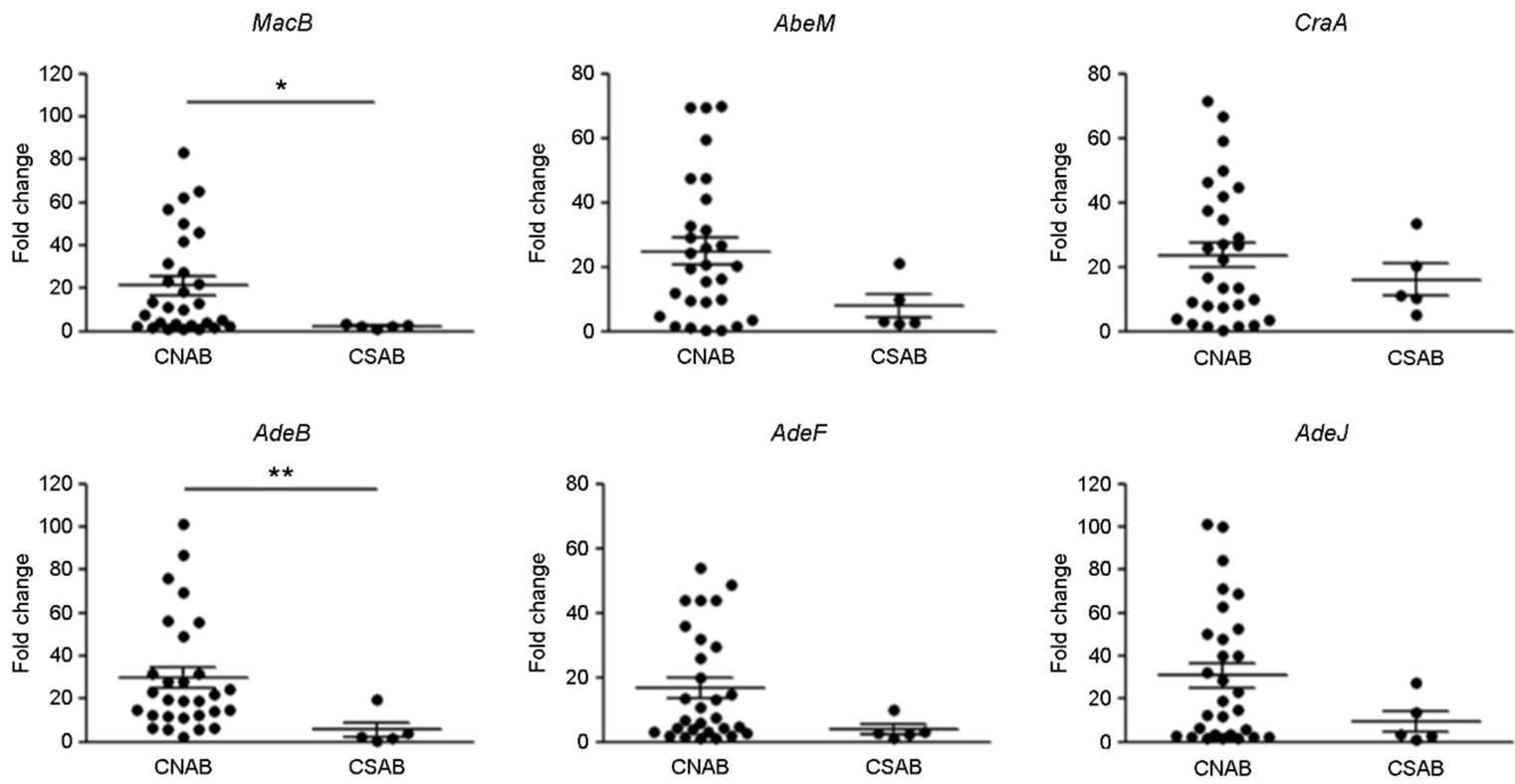

Fig. 1. Relationship between selective efflux pump gene expression and imipenem resistant isolates. The fold change mentioned are the average of all the test isolates when tested in triplicate. * and ** represents $P<0.05$ and $<0.005$. In carbapenem resistant isolates, the $a d e B$ $(P=0.0056)$ and $\operatorname{macB}(P=0.0462)$ genes were overexpressed.

A

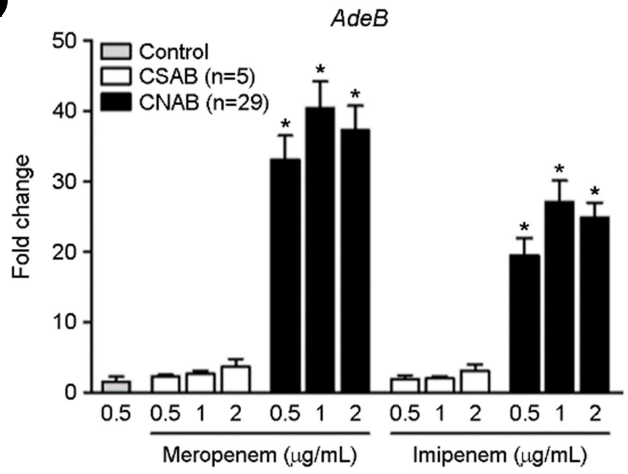

C

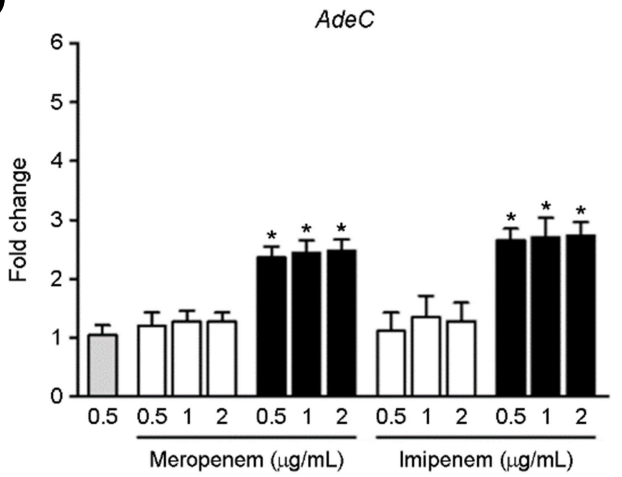

B

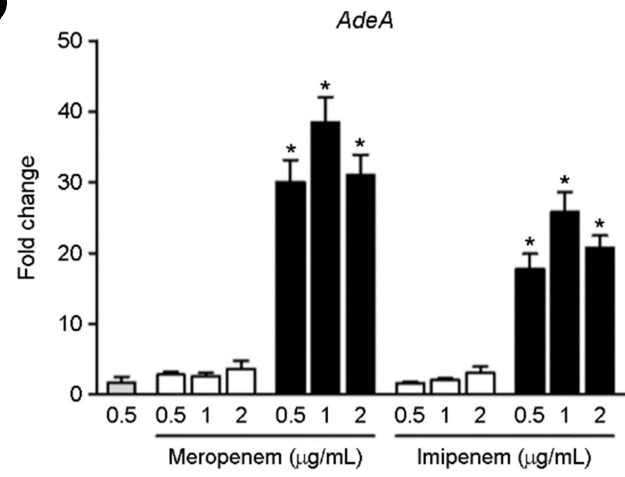

D

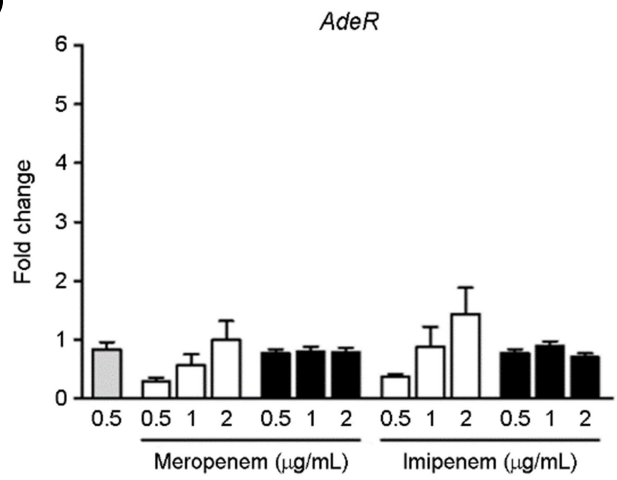

Fig. 2. Expression of adeB, adeA, adeC and adeR genes of test isolate under carbapenem stress relative to A. baumannii ATCC17978. (The value against each bar is the average fold change of all the test isolates) when tested in triplicate. Control = A. baumannii ATCC17978 in normal condition without stress. The value against each bar is the average fold change of all the test isolates). The fold change mentioned are the average of all the test isolates when tested in triplicate. * represents $P<0.01$ 
(macB, abeM, craA, adeB, adeF, and adeJ) and antimicrobial resistance to carbapenem antibiotics, $\operatorname{ade} B(P=0.0056)$ and $\operatorname{mac} B(P=0.0462)$ expression was significantly different. Among the efflux genes investigated, the clinical isolates with the highest expression of $a d e B$ were resistant to meropenem and imipenem compared to the susceptible isolates.

\section{Assessment of AdeABC efflux pump related genes}

The expression of the AdeABC efflux pump related genes following concentration-dependent meropenem or imipenem stress was also investigated. An increase in the expression of $a d e B$ caused a substantial reduction above a certain concentration ( $1 \mu \mathrm{g} / \mathrm{mL}$ ) was noticed (Fig. 2A). A similar response to imipenem was observed with adeA as well. The expression of adeA increased up to a certain concentration and a decline was observed at higher concentrations (Fig. 2B). In the case of adeC, hyperexpression was detected against both meropenem and imipenem stress irrespective of concentrations (Fig. 2C). The expression of adeS did not show any concentration-specific pattern (data not shown). Exposure of all the isolates to imipenem stress resulted in an increase in the expression of adeR in comparison to $A$. baumannii ATCC17978 under normal conditions (Fig. 2D). mRNA sequencing results show that seven isolates differed from the other isolates in the adeR and adeS regions. Three isolates had a point mutation in adeR (Pro116Leu). Four isolates had two mutations located in the adeR (Pro116Leu) and adeS (Thr153Met).

\section{DISCUSSION}

Preventing the spread of multi-drug resistant (MDR) bacteria is a major public health concern. Mutations responsible for the mechanisms underlying the emergence of the MDR phenotypes have been well characterized (Nikaido, 2009). However, there are a number of mechanisms by which the bacteria survive antibiotics treatment. Indeed, a possible mechanism of multi-drug resistance is the overexpression of efflux pump systems, which are membrane proteins eliminating toxic substances from the cell (Grkovic et al., 2001). Efflux pumps such as MacAB-TolC and AdeABC are essential for bacterial survival and colonization/virulence over the course of infection, especially when the pathogens are attacked by toxic substances or attached to the host (Okada et al., 2017; Coyne et al., 2011). Previous studies on wholegenome pyrosequencing of epidemic multidrug-resistant $A$. baumannii strains have shown that active extrusion by efflux pumps (including members of the MFS family) is considered an important factor in the multi-resistance phenotype of $A$. baumannii (Smith et al., 2007; Iacono et al., 2008). In the present study, a comparison of the relationship between gene expression of selected efflux pumps including $a d e B$, $m a c B$, abeM, craA, adeF, and adeJ genes and carbapenem antibiotics was investigated. A strong correlation between carbapenem resistance and over-expression of $a d e B$ was observed. The AdeABC efflux pump system is the main RND-type system in A. baumannii (Coyne et al., 2011), which is a transporter protein known to be responsible for efflux of aminoglycosides. There are few data available on the characterization of efflux pumps and the role between these elements in carbapenem resistance exhibited by $A$. baumannii. Our results provide more insight on some of the mechanisms underlying the increased pump activity due to overexpression.

The current study also investigated the role of efflux pump-mediated resistance and the transcriptional response of the adeA, adeB, adeC, adeR, and adeS genes from the clinical isolates of $A$. baumannii to a concentration gradient of carbapenem stress. The adeABC efflux pump operon consists of the ade $A$ (membrane fusion), adeB (multidrug transporter), and ade $C$ (outer membrane) genes. Furthermore, there are two regulatory genes, adeS (sensor kinase) and adeR (regulator), which are involved in controlling the expression of the AdeABC pump (Coyne et al., 2011; Marchand et al., 2004). It was demonstrated that the cleaved AdeS protein was constitutively produced and stimulated the expression of the AdeABC efflux pump through interaction with AdeR (Sun et al., 2014). In our study, the carbapenem resistant isolates under imipenem or meropenem stress showed much higher expression of adeA, adeB, and adeC in comparison to A. baumannii ATCC17978 under normal conditions, whereas none of the isolates showed an increase in the expression of adeR to carbapenem antibiotics. The study established that the AdeABC efflux pump is a related 
antibiotic resistance determinant in the bacterial pathogen A. baumannii and plays an important role in conferring resistance to the carbapenem group of antibiotics.

Our study also used a molecular approach to block efflux via EPIs and evaluate whether antibiotic sensitivity was restored in these clinical isolates. EPIs are drugs that can suppress resistance by blocking bacterial pumps and the release of certain antibiotics (Ni et al., 2016). The inhibitors $\mathrm{PA} \beta \mathrm{N}$ and CCCP, which are highly effective against efflux mechanisms, are known to have potent broad-spectrum activity in several studies (Baugh et al., 2014; Pagès et al., 2005). When CCCP or PA $\beta N$ was added in our study, no significant decline in the MICs of carbapenem was observed in both $\mathrm{CNAB}$ and $\mathrm{CSAB}$ isolates. These results further showed that $\mathrm{CCCP}$ and $\mathrm{PA} \beta \mathrm{N}$ may be ineffective for carbapenem antibiotics due to intrinsic resistance against carbapenem antibiotics in A. baumannii species (Peleg et al., 2007).

In conclusion, we have documented the emergence and wide dissemination of carbapenem-resistant $A$. baumannii isolates in a Busan university hospital in Korea. Effluxmediated mechanisms, including high levels of AdeABC expression, seem to play an important role in reduced carbapenem susceptibility, while active MacAB-TolC, CraA, AdeIJK, AdeFGH, and AbeM did not appear to be major contributors to carbapenem resistance. Both of the putative EPIs, PA $\beta \mathrm{N}$ and CCCP, could partially reverse the resistance to carbapenem, although neither had significant effects. It is speculated that antimicrobial agents have various binding sites in the efflux pumps; so, these EPIs can be activated in diverse manners (Schumacher et al., 2006). This study focused on analyzing the correlation between the overexpression of the active efflux pumps and the level of carbapenem resistance in multi-resistant $A$. baumannii isolates. The obtained results revealed that the AdeABC efflux pump correlates with carbapenem resistance. Therefore, we confirmed that the expression of AdeABC efflux pump associated genes, which are involved in the carbapenem resistance mechanism of $A$. baumannii, are important. Our results also indicate that adeABC and adeR expression affects carbapenem resistance, but the expression of these genes alone is insufficient to completely account for the resistance. Further studies should be conducted country-wide to provide specific guidelines for reducing the prevalence of efflux pump activation in multi-drug resistant $A$. baumannii clinical isolates

\section{ACKNOWLEDGEMENT}

This work was done with research funds from the Ministry Education, Republic of Korea through the National Research Foundation (NRF) grant funded by the South Korea government (2019R1C1C1004820) and Catholic University of Pusan (2019).

\section{CONFLICT OF INTEREST}

The authors declare that they have no conflict of interest.

\section{REFERENCES}

Amabile-Cuevas CF, Arredondo-Garcia JL, Cruz A, Rosas I. Fluoroquinolone resistance in clinical and environmental isolates of Escherichia coli in Mexico City. J Appl Microbiol. 2010. 108: 158-162.

Bae IK, Jeong SH, Lee K. Carbapenem-resistant Acinetobacter baumannii. Korean J Clin Microbiol. 2012. 15: 1-8.

Batirel A, Balkan II, Karabay O, Agalar C, Akalin S, Alici O, et al. Comparison of colistin-carbapenem, colistin-sulbactam, and colistin plus other antibacterial agents for the treatment of extremely drug-resistant Acinetobacter baumannii bloodstream infections. Eur J Clin Microbiol Infect Dis. 2014. 33: 13111322.

Baugh S, Phillips CR, Ekanayaka AS, Piddock LJ, Webber MA Inhibition of multidrug efflux as a strategy to prevent biofilm formation. J Antimicrob Chemother. 2014. 69: 673-681.

Blair JM, Webber MA, Baylay AJ, Ogbolu DO, Piddock LJ. Molecular mechanisms of antibiotic resistance. Nat Rev Microbiol. 2015. 13: 42-51.

Chan BCL, Han XQ, Lui SL, Wong CW, Wang TB, Cheung DW, et al. Combating against methicillin-resistant Staphylococcus aureus-two fatty acids from purslane (Portulaca oleracea L.) exhibit synergistic effects with erythromycin. J Pharm Pharmacol. 2015. 67: 107-116.

Coyne S, Courvalin P, Périchon B. Efflux-mediated antibiotic resistance in Acinetobacter spp. Antimicrob Agents Chemother. 2011. 55.3: 947-953

Fernandez L, Hancock R. Adaptive and mutational resistance: role 
of porins and efflux pumps in drug resistance. Clin Microbiol Rev. 2013. 25: 661-681.

Grkovic S, Brown MH, Skurray RA. Transcriptional regulation of multidrug efflux pumps in bacteria. Semin Cell Dev Biol. 2001. 12: 225-237.

Hsu AJ, Tamma PD. Treatment of multidrug-resistant gram-negative infections in children. Clin Infect Dis. 2014. 58: 1439-1448.

Iacono M, Villa L, Fortini D, et al. Whole-genome pyrosequencing of an epidemic multidrug-resistant Acinetobacter baumannii strain belonging to the European clone II group. Antimicrob Agents Chemother. 2008. 52: 2616-2625.

Jassim KA, Ghaima KK, Saadedin SMK. AdeABC Efflux pump genes in multidrug resistant Acinetobacter baumannii isolates. Avicenna J Clin Microbiol Infect. 2016. 3: 40898.

Kim JY, Kim SH, Jeon SM, Park MS, Rhie HG, Lee BK. Resistance to fluoroquinolones by the combination of target site mutations and enhanced expression of genes for efflux pumps in Shigella flexneri and Shigella sonnei strains isolated in Korea. Clin Microbiol Infec. 2008. 14: 760-765.

Kim YA, Park YS. Epidemiology and treatment of antimicrobialresistant gram-negative bacteria in Korea. Korean J Intern Med. 2018. 33: 247-255.

Lin MF, Lin YY, Tu CC, Lan CY. Distribution of different efflux pump genes in clinical isolates of multidrug-resistant Acinetobacter baumannii and their correlation with antimicrobial resistance. J Microbiol Immunol Infect. 2017. 50: 224-231.

Livak KJ, Schmittgen TD. Analysis of relative gene expression data using real-time quantitative PCR and the 2(-Delta Delta C(T)) Method. Methods. 2001. 25: 402-408.

Marchand I, Damier-Piolle L, Courvalin P, Lambert T. Expression of the RND-type efflux pump AdeABC in Acinetobacter baumannii is regulated by the AdeRS two-component system. Antimicrob Agents Chemother. 2004. 48: 3298-3304.

Ni W, Li Y, Guan J, Zhao J, Cui J, Wang R, et al. Effects of efflux pump inhibitors on colistin resistance in multidrug-resistant gram-negative bacteria. Antimicrob Agents Chemother. 2016. 60: 3215-3218.

Nikaido H. Multidrug Resistance in Bacteria. Annu Rev Biochem. 2009. 78: 119-146.

Okada U, Yamashita E, Neuberger A, Morimoto M, van Veen HW, Murakami S. Crystal structure of tripartite-type ABC transporter MacB from Acinetobacter baumannii. Nat Commun. 2017. 8: 1336.

Ostad Asadolah-Malayeri H, Hakemi-Vala M, Davari K. Role of AdeRs and OXA23 genes among imipenem resistant Acineto- bacter baumannii isolates from two hospitals of Tehran, Iran. Iran J Pathol. 2016. 11: 345.

Pagès JM, Masi M, Barbe J. Inhibitors of efflux pumps in Gramnegative bacteria. Trends Mol Med. 2005. 11: 382-389.

Park SY, Lee EJ, Kim T, Yu SN, Park KH, Lee MS, et al. Early administration of appropriate antimicrobial agents to improve the outcome of carbapenem-resistant Acinetobacter baumannii complex bacteraemic pneumonia. Int J Antimicrob Agents. 2018. 51: 407-412.

Peleg AY, Adams J, Paterson DL. Tigecycline efflux as a mechanism for nonsusceptibility in Acinetobacter baumannii. Antimicrob Agents Chemother. 2007. 51: 2065-2069.

Pogue JM, Neelakanta A, Mynatt RP, Sharma S, Lephart P, Kaye KS. Carbapenem-resistance in gram-negative bacilli and intravenous minocycline: an antimicrobial stewardship approach at the Detroit Medical Center. Clin Infect Dis. 2014. 59: S388S393.

Qiu ZQ, Zhu LJ, Hou PF. Distribution of carbapenemases and efflux pump in carbopenems-resistance Acinetobacter baumannii. Peer J Preprints. 2016. 4: e2655v1.

Roca I, Marti S, Espinal P, Martetínez P, Gibert I, Vila J. CraA, a major facilitator superfamily efflux pump associated with chloramphenicol resistance in Acinetobacter baumannii. Antimicrob Agents Chemother. 2009. 53: 4013-4014.

Rosales-Reyes R, Gayosso-Vazquez C, Fernandez-Vazquez JL, Jarillo-Quijada MD, Rivera-Benitez C, Santos-Preciado JI, et al. Virulence profiles and innate immune responses against highly lethal, multidrug-resistant nosocomial isolates of Acinetobacter baumannii from a tertiary care hospital in Mexico. PLoS One. 2017. 12: e0182899.

Schumacher A, Steinke P, Bohnert JA, Akova M, Jonas D, Kern WV. Effect of 1-(1-naphthylmethyl)-piperazine, a novel putative efflux pump inhibitor, on antimicrobial drug susceptibility in clinical isolates of Enterobacteriaceae other than Escherichia coli. J Antimicrob Chemother. 2006. 57: 344-348.

Shi WF, Jiang JP, Xu N, Huang ZM, Wang YY. Inhibitory effects of reserpine and carbonyl cyanide m-chloro-phenylhydrazone on fluoroquinolone resistance of Acinetobacter baumannii. Chin Med J. 2005. 118: 340-343.

Shigemura K, Osawa K, Kato A, Tokimatsu I, Arakawa S, Shirakawa T, et al. Association of overexpression of efflux pump genes with antibiotic resistance in Pseudomonas aeruginosa strains clinically isolated from urinary tract infection patients. J Antibiot. 2015. 68: 568.

Smith MG, Gianoulis TA, Pukatzki S, et al. New insights into 
Acinetobacter baumannii pathogenesis revealed by highdensity pyrosequencing and transposon mutagenesis, Genes De. 2007. 21: 601-614.

Srinivasan VB, Rajamohan G, Gebreyes WA. The role of AbeS, a novel efflux pump member of the SMR family of transporters, in resistance to antimicrobial agents in Acinetobacter baumannii. Antimicrob Agents Chemother. 2009. 53: 53125316.

Su XZ, Chen J, Mizushima T, Kuroda T, Tsuchiya T. AbeM, an $\mathrm{H}+$-coupled Acinetobacter baumannii multidrug efflux pump belonging to the MATE family of transporters. Antimicrob Agents Chemother. 2005. 49: 4362-4364.

Sun J, Deng Z, Yan A. Bacterial multidrug efflux pumps: Mechanisms, physiology and pharmacological exploitations. Biochem Biophys Res Commun. 2014. 453: 254-267.

Swick MC, Morgan-Linnell SK, Carlson KM, Zechiedrich L. Expression of multidrug efflux pump genes acrAB-tolC, $m d f A$, and norE in Escherichia coli clinical isolates as a function of fluoroquinolone and multidrug resistance. Antimicrob Agents Chemother. 2011. 55: 921-924.

Wayne PA, Clinical and Laboratory Standards Institute. Performance Standards for Antimicrobial Susceptibility Testing; Twenty-second Informational Supplement. CLSI Document. 2012. M100-S122.

Xing L, Barnie PA, Su Z, Xu H. Development of efflux pumps and inhibitors (EPIs) in A. baumannii. Clin Microbiol. 2014. 3: 135 .

https://doi.org/10.15616/BSL.2021.27.2.59

Cite this article as: $\mathrm{Ju} \mathrm{Y}$, Kim YJ, Chang CL, Choi GE, Hyun KY. Relationship between AdeABC Efflux Pump Genes and Carbapenem in Multidrug-resistant Acinetobacter baumannii. Biomedical Science Letters. 2021. 27: 59-68. 